\title{
Ultrasound validity in the measurement of knee cartilage thickness
}

\author{
E Naredo, ${ }^{1}$ C Acebes, ${ }^{2}$ I Möller, ${ }^{3}$ F Canillas, ${ }^{4} \mathrm{~J} J$ de Agustín, ${ }^{5}$ E de Miguel, ${ }^{6}$ E Filippucci, ${ }^{7}$ \\ A lagnocco, ${ }^{8} \mathrm{C}$ Moragues, ${ }^{9} \mathrm{R}$ Tuneu, ${ }^{10} \mathrm{~J}$ Uson, ${ }^{11} \mathrm{~J}$ Garrido, ${ }^{12} \mathrm{E}$ Delgado-Baeza, ${ }^{13}$ \\ I Sáenz-Navarro ${ }^{14,15}$
}

${ }^{1}$ Rheumatology, Hospital Severo Ochoa, Madrid, Spain:

${ }^{2}$ Rheumatology, Fundación Jiménez Díaz, Madrid, Spain;

${ }^{3}$ Rheumatology, Instituto Poal, Barcelona, Spain; ${ }^{4}$ Anatomy, Histology and Neuroscience, Orthopaedic Research Laboratory, Histology Laboratory A21, Faculty of Medicine, Autónoma University, Madrid, Spain; ${ }^{5}$ Rheumatology, Hospital Vall d'Hebron, Barcelona, Spain;

${ }^{6}$ Rheumatology, Hospital La Paz, Madrid, Spain; ${ }^{7}$ Rheumatology, Universitá Politecnica delle

Marche, Ancona, Italy;

${ }^{8}$ Rheumatology, Sapienza

University, Rome, Italy;

${ }^{9}$ Rheumatology, Hospital de

Bellvitge, Barcelona, Spain;

${ }^{10}$ Rheumatology, Hospital de

Manresa, Barcelona, Spain;

${ }^{11}$ Rheumatology, Hospital de

Móstoles, Madrid, Spain;

${ }^{12}$ Methodology, Autónoma

University, Madrid, Spain;

${ }^{13}$ Anatomy, Histology and

Neuroscience, Orthopaedic

Research Laboratory, Histology

Laboratory A21, Faculty of

Medicine, Autónoma University,

Madrid, Spain; ${ }^{14}$ Human

Anatomy and Embryology,

Faculty of Medicine, University

of Barcelona, Barcelona, Spain;

${ }^{15}$ Orthopedic Surgery,

Fundación Hospital Espíritu

Santo, Santa Coloma de

Gramanet, Barcelona, Spain

Correspondence to:

E Naredo, Calle Arturo Soria

259, $4^{\circ}$ A, 28033 Madrid, Spain; enaredo@ser.es

Accepted 26 July 2008

Published Online First

6 August 2008

\section{ABSTRACT}

Objective: To assess the multiexaminer reproducibility and the accuracy comparing with cadaver anatomic specimens of ultrasound (US) measurement of femoral articular cartilage (FAC) thickness.

Methods: In 8 flexed cadaver knees, FAC thickness was blindly, independently and consecutively measured twice by 10 rheumatologists at the lateral condyle (LC), medial condyle (MC) and intercondylar notch (IN) with US. After the US measurements, the knees were dissected. Articular cartilage integrity was evaluated macroscopically in the femoral condyles. FAC thickness was blindly measured in the specimens using a stereoscopic magnifying loupe and a digitised image software. Interexaminer and intraexaminer reliability of US FAC thickness measurement and agreement between US and anatomic measurements were assessed by estimating the intraclass correlation coefficient (ICC).

Results: Interexaminer ICCs were higher than 0.90 for MC $(p<0.001)$ and IN $(p<0.001)$ and higher than 0.75 for LC $(p<0.01)$. Mean intraexaminer ICCs were 0.832 for MC ( $p<0.001), 0.696$ for LC $(p<0.001)$ and, 0.701 for IN $(p<0.001)$. Agreement between US and anatomic FAC thickness measurements was good for MC (ICC 0.719; $p=0.020)$ and poor for $L C(p=0.285)$ and $I N$ $(p=0.332)$. Bland-Altman analysis showed that the difference between US and anatomic values was considerably high in the one knee with severely damaged FAC. After eliminating this knee from the analysis, ICCs were $0.883(p<0.001)$ for $M C, 0.795(p=0.016)$ for $L C$ and 0.732 for IN $(p=0.071)$.

Conclusion: US demonstrated a good reproducibility in FAC thickness measurement by multiple examiners. In addition, US FAC thickness measurement was accurate in normal to moderately damaged cartilage.

Osteoarthritis (OA) is the most common joint disease. It is the most frequent cause of rheumatic complaints and a relevant public health problem. $\mathrm{OA}$ is characterised by focal degeneration and progressive loss of articular cartilage in the involved joints.

Successful assessment of $O A$ progression and therapeutic response to interventions that could control the course of this disease depend on establishing objective methods for monitoring articular cartilage damage. Before assessing the responsiveness of any method, it is necessary to demonstrate its validity and reproducibility.

For many years, clinical studies on drug interventions have used a variety of analogue scales for measuring pain and joint function in symptomatic OA without assessing the effect of treatment on structural changes caused by the disease or the role of therapy in preventing cartilage degradation. Pain scales may only reflect the analgesic properties of a given intervention and, because articular cartilage lacks pain nerve fibres, pain in OA joints probably comes from other anatomic structures such as subchondral bone, synovium, tendons and muscles, making these assessments indirect. ${ }^{1}$

The availability of valid, reproducible and responsive imaging modalities to detect and quantify cartilage damage is necessary for improving assessment of OA progression and response to treatment. Because articular cartilage lesions are always found in $\mathrm{OA}$, a valid technique for monitoring the disease should be able to evaluate morphostructural features of articular cartilage.

Plain radiography has been considered the gold standard for assessing joint damage in OA. However, this technique shows only indirect signs of articular cartilage involvement and is not sensitive to minor changes in cartilage thickness. ${ }^{1-3}$ Arthroscopy, MRI and ultrasound (US) allow for direct visualisation of articular cartilage. Arthroscopy has proven reliability and sensitivity to change in its assessment. ${ }^{4}$ However, only the cartilage surface can be evaluated, and its invasive nature limits the use of this technique as a routine assessment tool in OA. Several studies have demonstrated the accuracy and reliability of MRI for evaluating structure in OA. ${ }^{25-8}$ Advantages of MRI include its non-invasiveness, multiplanar capability and excellent soft tissue contrast. However, MRI is expensive, time consuming and not widely available for routine use.

High-resolution US is an accurate, inexpensive, readily accepted by patients and non-invasive method for imaging the musculoskeletal system. Articular cartilage can be visualised directly by US at different peripheral joints, including the knee, elbow, wrist, shoulder, tibiotalar and metacarpophalangeal joints. It appears as a homogeneous anechoic band due to the high water content between the bony cortex and the soft tissues delimited by two sharp and hyperechoic interfaces. The lack of echoes and the sharpness of the synovial space-cartilage and cartilage-bone interfaces are the principal features of articular cartilage in healthy subjects. Nearly complete lack of articular cartilage can be observed in patients with late $\mathrm{OA} .^{9}$

Among peripheral joints, the knee is the most commonly involved joint in OA. ${ }^{10}{ }^{11}$ It is possible to expose to US a significant portion of the femoral 
articular cartilage by hyperflexing the knee. Various studies have shown that femoral articular cartilage (FAC) thickness can be measured by US ${ }^{12-18}$ with good intraobserver and interobserver reliability between two examiners. ${ }^{15-18}$

However, there is a paucity of data on the criterion validity of US measurement of the thickness of human articular cartilage ${ }^{18}$ and there are no studies on US reliability between multiple examiners of articular cartilage thickness measurement.

The purpose of this study was to assess the multiexaminer reproducibility of US in the measurement of FAC thickness. In addition, we investigated the criterion validity of US FAC thickness measurement using cadaver anatomic specimens as gold standard.

\section{METHODS}

Eight fresh-frozen human cadaver knees (four right knees, four left knees) from five cadavers (age of death range 76-89 years) were thawed at room temperature. The knees were flexed maximally in order to make a great part of the weight-bearing surface of the femoral condyles accessible to US assessment. The grade of knee flexion ranged from $124^{\circ}$ to $141^{\circ}$. The fully flexed knees were fastened during the study using cadaveric specimen supports (Human Anatomy Department, Faculty of Medicine, University of Barcelona, Barcelona, Spain).

\section{US investigation}

A total of 10 rheumatologists, expert in musculoskeletal US, participated in the interexaminer and intraexaminer reliability study. The knees were scanned with two identical real-time US machines (Logiq 9, Wauwatosa, Wisconsin, USA) using multifrequency linear array transducers. The settings of both US machines were adjusted and standardised just before the study began. These settings resulted in a frequency of $14 \mathrm{MHz}$, dynamic range of $72 \mathrm{~dB}$, gain of $37 \mathrm{~dB}$ and depth of $28 \mathrm{~mm}$.

The length of the transducer footprint, which corresponds to the distance between the lateral edges of the US screen measured with the machine's electronic calliper, was $39 \mathrm{~mm}$. In order to standardise FAC thickness measurement, three vertical parallel lines were drawn on a transparent sheet put against the screen of each US machine, one line at the centre of the screen and two lines midway between the centre and the lateral edges of the screen.

Before the start of the multiexaminer reliability study, one of the US investigators (CA) placed the transducer transversely to the leg just above the superior margin of the patella at each knee. When the midpoint of the intercondylar notch was imaged at the centre of the machine screen, he marked two lines in the cadaver knee skin, each one at the midpoint of the lateral edges of the transducer and perpendicular to them using a scalpel. He oriented the probe perpendicular to bone surface to optimise the FAC US image. The angle between the transducer position and the horizontal position was recorded for each knee. These angles ranged from $0^{\circ}$ to $10^{\circ}$.

The US multiexaminer reliability study was carried out in 1 day. It took $9 \mathrm{~h}$ divided into two sessions, a 4.5-h morning session and a 4.5 -h afternoon session. Four knees were randomly assigned to the morning session and four knees to the afternoon session for the US investigation. The knees were blindly, independently and consecutively scanned by the 10 US experts in two rounds in a random fashion during each session. The two US examinations of each knee were performed by each investigator with a minimum interval of $2 \mathrm{~h}$.
FAC thickness was measured by US in the lateral condyle (LC), medial condyle (MC) and intercondylar notch (IN) with the transducer placed transversely to the leg just above the upper pole of the patella between the two lines marked on the cadaver skin (fig 1). The points where the three lines drawn on the transparent sheets intersected the bone-cartilage interface at the LC, MC and IN were taken as reference US measurement points. The US machine allowed measurements of thousandths of mm. FAC thickness was measured perpendicular to the bonecartilage interface at the three reference points.

Each US expert was given a maximum of 4 min for US imaging and measuring FAC thickness and filling in a standardised sheet with the measures of FAC thickness. A statistician (JG) was at hand during the US study to codify each knee, ${ }^{1-8}$ to assign each expert to the corresponding knee during each round, to check and record the time of US scanning and to receive the filled sheets. An application specialist from the US company was present to codify each knee assessment in the machine and to save all the FAC images produced by the 10 examiners during the study to the machine's hard drive.

\section{Anatomic investigation}

After the US measurements, the femoral condyles of each knee were perforated from the cadaver skin at the midpoints of the lines drawn at the lateral edges of the transducer with a drill directed perpendicularly to the orientation angle of the transducer. Then, the knees were dissected. Full-thickness $20-\mathrm{mm}$ slices of articular cartilage and underlying subchondral bone of the femoral condyles were cut and fixed in 6\% buffered formalin solution. These samples were sectioned carefully in the transverse plane along the line that joined the two holes made in the femoral condyles. A sharpened blade was used to cut the specimens in order to avoid damage to the cartilage.

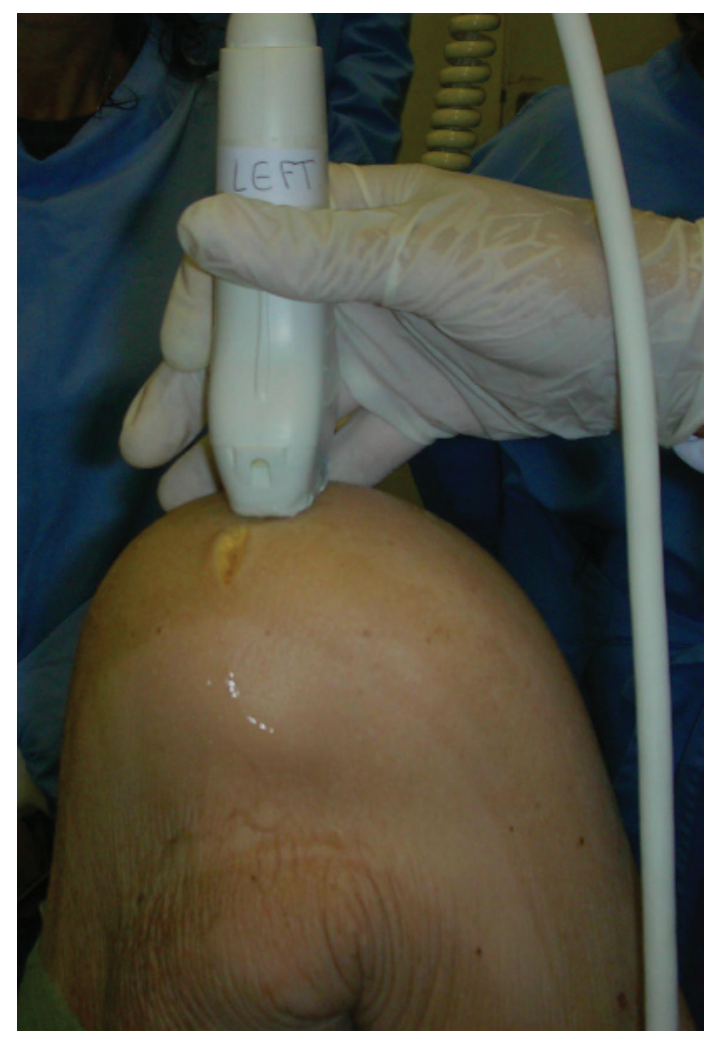

Figure 1 Transducer placement for measuring femoral articular cartilage thickness. 
Surface and full-thickness articular cartilage integrity were evaluated macroscopically in the anatomic sections of the femoral condyles by one anatomist (EDB), highly experienced in cartilage research, blinded to the US data. The femoral condyles were classified as normal, mild lesion, moderate lesion and severe lesion according to the macroscopic appearance of their articular cartilage.

Anatomic reference points corresponding to the US measuring points at the midpoint of the IN and $9.75 \mathrm{~mm}$ apart at the LC and MC were marked in the anatomic sections. Each anatomic slice along with a measuring scale was optically visualised and digitised using a stereoscopic magnifying loupe (Ceti, Grimbergen, Belgium). FAC thickness was blindly, independently and consecutively measured twice by two sonographists (CA and EN) and one anatomist (FC). The two rounds of anatomic measurements were carried out by each investigator with a minimum interval of $2 \mathrm{~h}$. FAC thickness was measured perpendicular to the tidemark at the three anatomic reference points using ImageJ $\mathrm{V} 1.36 \mathrm{~b}$ software. (Wayne Rashband, National Institutes of Health, Washington DC, USA) (fig 2). FAC thickness measures were obtained within thousandths of $\mathrm{mm}$.

\section{Statistical analysis}

Intraexaminer reliability of US FAC thickness measurement and intraobserver reliability of anatomic FAC thickness measurement were evaluated by calculating the intraclass correlation coefficient (ICC) using a two-factor random model, with knee and examiner/observer as random factors. Interexaminer reliability of US FAC thickness measurement and interobserver reliability of anatomic FAC thickness measurement were assessed by estimating ICC for absolute agreement using a two-factor random model, with knee and examiner/observer as random factors. Agreement between US and anatomic FAC thickness measurements were evaluated by calculating ICC for absolute agreement using a two-factor mixed model with knee as random factor and technique as fixed factor. All ICC were calculated with the statistical program SPSS V 14.0 (SPSS, Chicago, Illinois, USA). ICC values $>0.50$ were considered poor, $0.50-0.60$ moderate, $0.60-0.80$ good and $0.80-1$ excellent. The differences between US and anatomic values versus means of

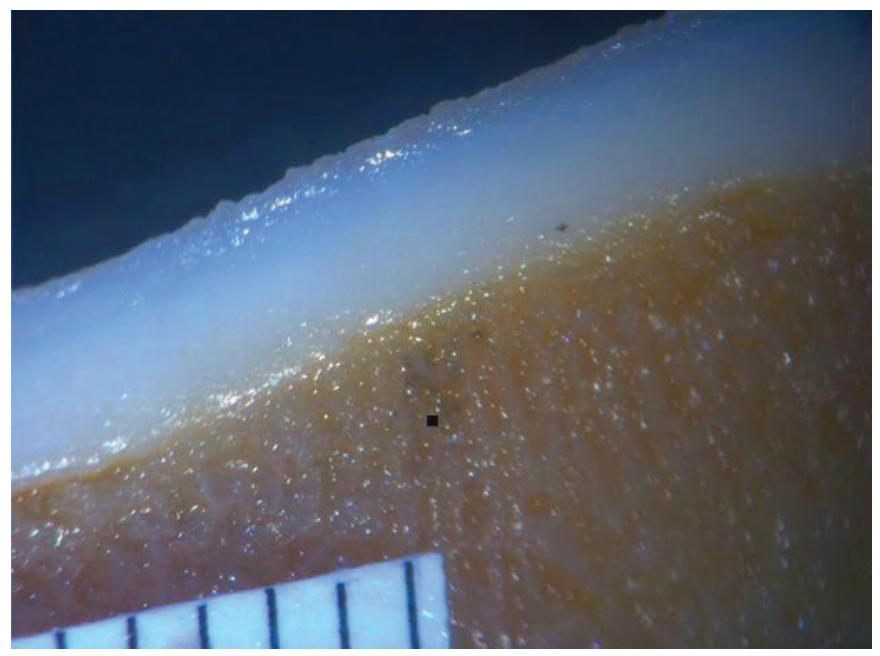

Figure 2 Digitised image of the medial condyle articular cartilage obtained with the stereoscopic magnifying loupe in an anatomic specimen.
US and anatomic values were plotted according to BlandAltman for disaggregated visual analysis of agreement.

\section{RESULTS}

In all cadaver knees the cartilage of the femoral condyles and intercondylar notch was imaged by US as an anechoic band having hyperechoic anterior and posterior interfaces, with the cartilage-bone interface appearing thicker than the synovial space-cartilage interface. Both interfaces showed variable sharpness in the different femoral condyles examined (fig 3A,B).

The anatomic sample where US measurements had been made corresponded to weight-bearing FAC at the level of the trochlea-condylar sulcus, which is located between the weightbearing femoropatellar and femorotibia cartilage. In the anatomic sections, sharpness of the tidemark and the superficial margin of femoral articular cartilage varied considerably between and within the femoral condyles (fig 2).
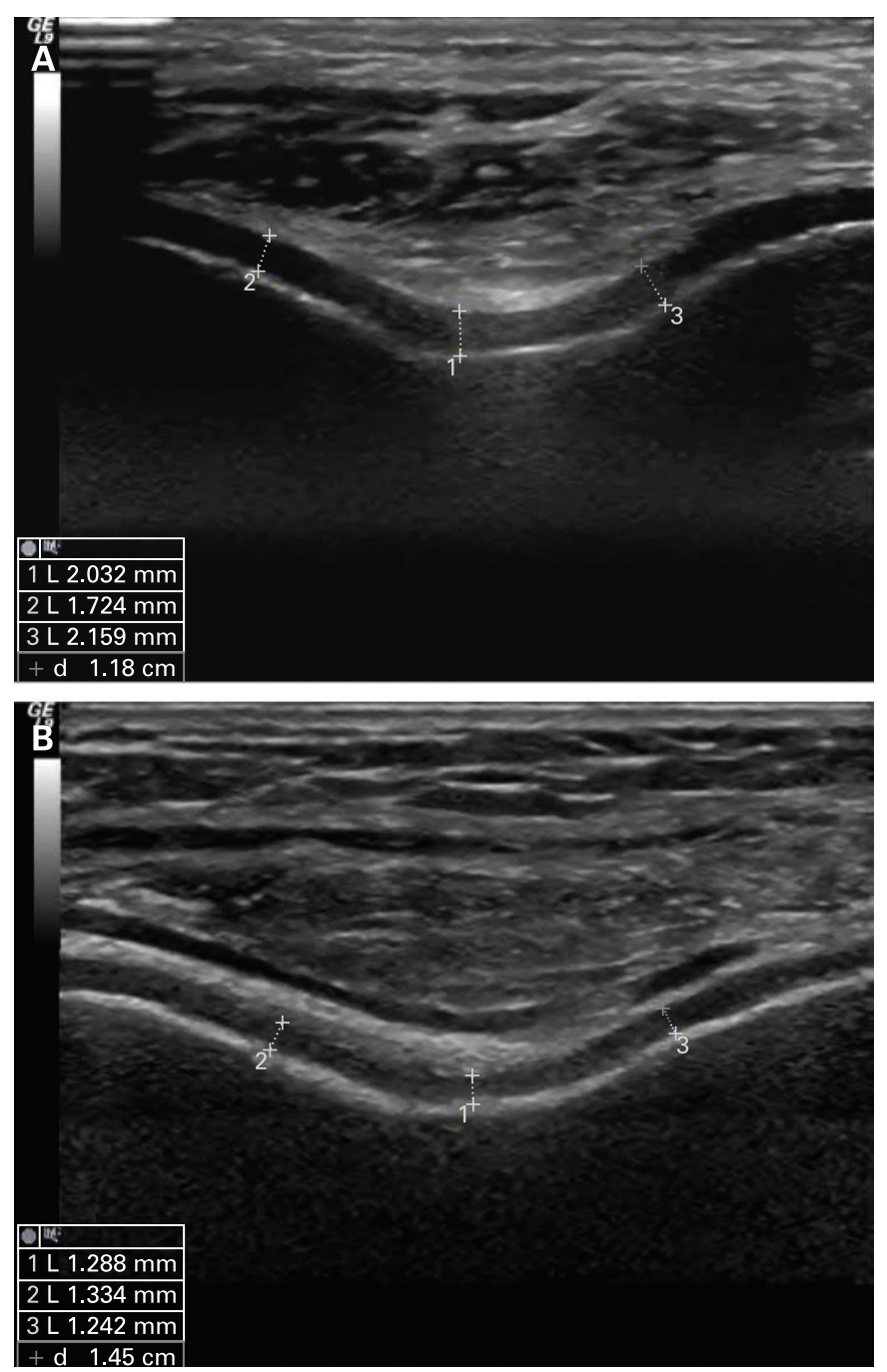

Figure 3 Ultrasound transverse images of the femoral articular cartilage with lateral condyle, intercondylar notch and medial condyle cartilage thickness measures. $(A)$ is from knee 1, which was classified as normal in macroscopic assessment. Articular cartilage shows hyperechoic sharply defined interfaces. $(B)$ is from knee 2, which was classified as severely damaged in macroscopic assessment. Articular cartilage appears thinner and shows less defined interfaces than knee 1. 
Mean (SD) US measures of FAC thickness were 1.771 (0.391) $\mathrm{mm}$ (range 1.054-2.964) at the LC, $1.845(0.428) \mathrm{mm}$ (range 0.991-3.133) at the MC and 1.893 (0.536) $\mathrm{mm}$ (range $0.799-4.113)$ at the IN. Mean (SD) anatomic measures of FAC thickness were $2.036(0.322) \mathrm{mm}$ (range 1.508-2.792) at the LC, $2.171(0.515) \mathrm{mm}$ (range 1.115-3.182) at the $\mathrm{MC}$ and 2.196 (0.522) $\mathrm{mm}$ (range 1.550-3.161) at the IN.

Macroscopic assessment of FAC resulted normal in four femoral condyles (LC and MD from knee 1, LC from knee 3 and $\mathrm{MC}$ from knee 5), mild lesion of the FAC in seven femoral condyles (MC from knee 3, LC from knee 4, LC from knee 5, LC from knee 6, LC from knee 7 and both condyles from knee 8), moderate lesion in three femoral condyles (MC from knee 4, $\mathrm{MC}$ from knee 6 and MC from knee 7) and severe lesion in two femoral condyles (both condyles from knee 2).

\section{Reliability of US measurement of femoral articular cartilage thickness}

Interexaminer and intraexaminer ICCs for US FAC thickness measurement are displayed in table 1. Interexaminer ICCs were higher than 0.90 for $\mathrm{MC}(\mathrm{p}<0.001)$ and IN $(p<0.001)$. Interexaminer ICC for LC was excellent in the first round of US assessment $(p<0.001)$ and good in the second round $(p=0.002)$.

Mean intraexaminer ICCs for US FAC thickness measurement were 0.832 (95\% confidence interval (CI) 0.737 to 0.892 ) for the MC, 0.696 (95\% CI 0.526 to 0.805 ) for the LC and 0.701 ( $95 \%$ CI 0.533 to 0.808$)$ for the IN. All of them were significant $(\mathrm{p}<0.001)$

\section{Reliability of anatomic measurement of femoral articular cartilage thickness}

Interobserver and intraobserver ICCs for anatomic FAC thickness measurement are shown in table 1. Interobserver ICCs were higher than 0.95 for the MC, LC and IN $(p<0.001)$. Mean intraobserver ICCs were 0.968 (95\% CI 0.925 to $0.986 ; p<0.001$ ) for the MC, 0.963 (95\% CI 0.914 to $0.984 ; \mathrm{p}<0.001)$ for the LC and 0.979 (CI 0.952 to $0.991 ; p<0.001$ ) for the IN.
Agreement between US and anatomic measurements of femoral articular cartilage thickness

Agreement between US and anatomic FAC thickness measurements was good for the MC (ICC $0.719 ; 95 \%$ CI -0.279 to $0.943 ; p=0.020$ ) and poor for the LC (ICC $0.284 ; 95 \%$ CI -0.895 to $0.826 ; p=0.285$ ) and IN (ICC $0.267 ; 95 \%$ CI -1.620 to $0.841 ; p=0.332$ ). Figure 4 displays differences between FAC thickness US and anatomic measures at the LC, IN and MC. Bland-Altman analysis revealed that most anatomic measures were higher than the corresponding US measures. Mean differences between US and anatomic measures were $-0.326 \mathrm{~mm} \quad(95 \%$ CI -0.763 to 0.111$)$ for the $\mathrm{MC}$, $-0.266 \mathrm{~mm}(95 \%$ CI -0.711 to 0.180$)$ for the LC and $-0.303 \mathrm{~mm}(95 \% \mathrm{CI}-0.884$ to 0.278$)$ for the IN. A noteworthy finding was the considerably higher difference between US and anatomic values for the LC, MC and IN from knee 2 when compared with the other knees. As knee 2 showed the most severely damaged articular cartilage in both condyles, it was eliminated from the analysis.

Bland-Altman analysis of seven knees showed a marked decrease in mean differences between US and anatomic measures and narrowing in 95\% CIs. Mean differences were $-0.225 \mathrm{~mm}(95 \% \mathrm{CI}-0.568$ to 0.119$)$ for the MC, $-0.152 \mathrm{~mm}$ (95\% CI -0.470 to 0.165$)$ for the LC and $-0.130 \mathrm{~mm}(95 \% \mathrm{CI}$ -0.600 to 0.340 ) for the IN. ICCs resulted from seven knees are shown in table 2. Agreement was excellent for MC cartilage thickness (ICC $0.883 ; 95 \%$ CI 0.045 to 0.981 ) and good for LC (ICC 0.795 ; $95 \%$ CI -0.097 to 0.964 ) and IN (ICC 0.732; 95\% CI -0.460 to 0.954 ) cartilage thickness. ICC was significant for LC and $\mathrm{MC}$ cartilage thickness. measurements Limits of agreement were -0.665 to $0.205 \mathrm{~mm}$ for the $\mathrm{MC},-0.520$ to $0.214 \mathrm{~mm}$ for the LC and -0.935 to $0.675 \mathrm{~mm}$ for the IN.

\section{DISCUSSION}

In vivo articular cartilage thickness measurement is an important marker of structural joint damage in $\mathrm{OA}$ and inflammatory arthritis. In addition, assessment of cartilage damage is important for monitoring disease progression and potentially evaluating therapeutic response.

Table 1 Reliability of ultrasound (US) and anatomic measurement of femoral articular cartilage (FAC) thickness

\begin{tabular}{|c|c|c|c|c|}
\hline Reliability & Measurement & Measuring point & ICC (95\% CI) & p Value \\
\hline \multirow[t]{6}{*}{ Interexaminer } & US FAC thickness, first round & Lateral condyle & $0.866(0.665$ to 0.968$)$ & $<0.001$ \\
\hline & US FAC thickness, first round & Medial condyle & $0.928(0.821$ to 0.983$)$ & $<0.001$ \\
\hline & US FAC thickness, first round & Intercondylar notch & $0.944(0.859$ to 0.987$)$ & $<0.001$ \\
\hline & US FAC thickness, second round & Lateral condyle & $0.754(0.348$ to 0.951$)$ & 0.002 \\
\hline & US FAC thickness, second round & Medial condyle & $0.965(0.908$ to 0.993$)$ & $<0.001$ \\
\hline & US FAC thickness, second round & Intercondylar notch & $0.932(0.819$ to 0.986$)$ & $<0.001$ \\
\hline \multirow[t]{3}{*}{ Intraexaminer } & US FAC thickness & Lateral condyle & $0.696(0.526$ to 0.805$)$ & $<0.001$ \\
\hline & US FAC thickness & Medial condyle & $0.832(0.737$ to 0.892$)$ & $<0.001$ \\
\hline & US FAC thickness & Intercondylar notch & $0.701(0.533$ to 0.808$)$ & $<0.001$ \\
\hline \multirow[t]{6}{*}{ Interobserver } & Anatomic FAC thickness, first round & Lateral condyle & $0.994(0.980$ to 0.999$)$ & $<0.001$ \\
\hline & Anatomic FAC thickness, first round & Medial condyle & $0.984(0.945$ to 0.996$)$ & $<0.001$ \\
\hline & Anatomic FAC thickness, first round & Intercondylar notch & 0.996 (0.986 to 0.999$)$. & $<0.001$ \\
\hline & Anatomic FAC thickness, second round & Lateral condyle & $0.963(0.874$ to 0.992$)$ & $<0.001$ \\
\hline & Anatomic FAC thickness, second round & Medial condyle & $0.959(0.860$ to 0.991$)$ & $<0.001$ \\
\hline & Anatomic FAC thickness, second round & Intercondylar notch & $0.982(0.940$ to 0.996$)$ & $<0.001$ \\
\hline \multirow[t]{3}{*}{ Intraobserver } & Anatomic FAC thickness & Lateral condyle & $0.963(0.914$ to 0.984$)$ & $<0.001$ \\
\hline & Anatomic FAC thickness & Medial condyle & $0.968(0.925$ to 0.986$)$ & $<0.001$ \\
\hline & Anatomic FAC thickness & Intercondylar notch & $0.979(0.952$ to 0.991$)$ & $<0.001$ \\
\hline
\end{tabular}

ICC, intraclass correlation coefficient. 


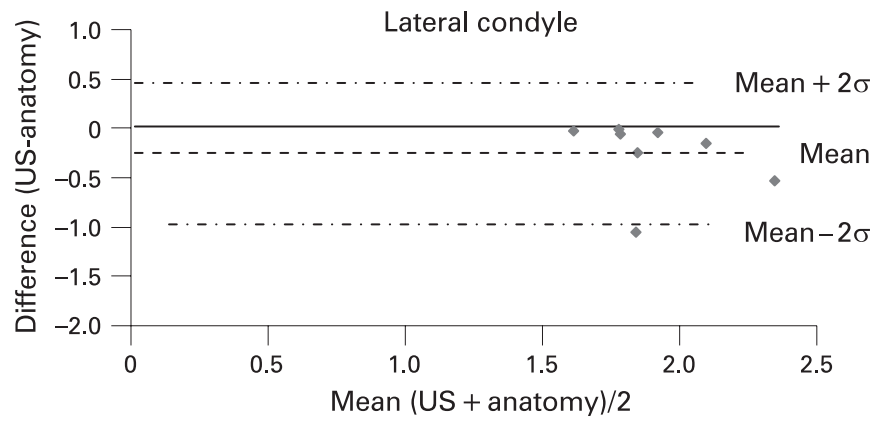

Intercondylar notch
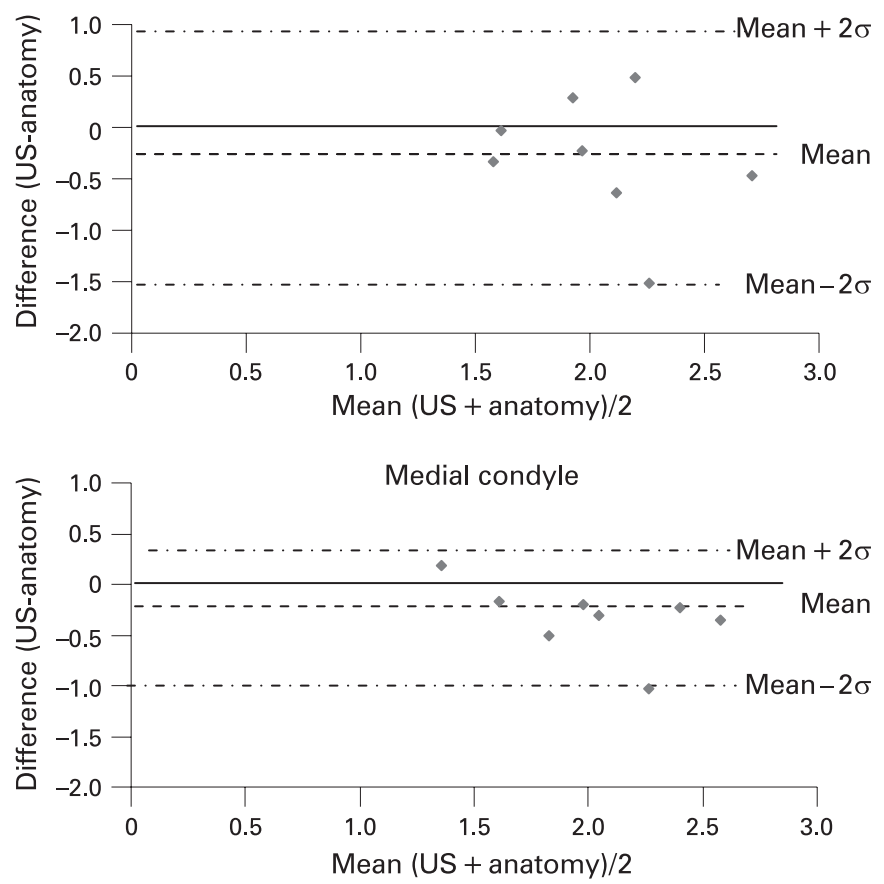

Figure 4 Agreement between ultrasonographic and anatomic measurements of femoral articular cartilage thickness. US, ultrasound.

Plain radiography is the imaging modality most frequently used for assessing $\mathrm{OA}$ and arthritic joint. However, plain radiographs cannot show the articular cartilage. In addition, this technique is limited by its inability to visualise synovial recesses, menisci and other soft tissues involved in the pathophysiology of OA.

US is widely available, safe, inexpensive and can give much information about articular cartilage, ${ }^{92-14}{ }^{19}$ synovitis, periarticular soft tissues and bony cortical abnormalities in peripheral OA joints. ${ }^{20-24}$ Nevertheless, US should be tested for accuracy, reproducibility and responsiveness before being used to measure and monitor articular cartilage thickness.

Among previous published studies, US measures of FAC thickness have been shown to correspond to anatomic values in in vitro studies in animals ${ }^{12}$ and in cadavers ${ }^{18}$ as well as showing acceptable reliability between two observers in in vivo studies in humans. ${ }^{15-17}$ Iagnocco et al demonstrated that cartilage thickness was diminished in patients with rheumatoid arthritis and OA compared to normal subjects. ${ }^{19}$ Experimental high frequency (25 MHz) US has demonstrated in vitro accuracy and reproducibility for measuring human femoral articular cartilage thickness. $^{25}$

We conducted this study for demonstrating the multiexaminer reproducibility and the accuracy of commercially
Table 2 Agreement between ultrasonographic and anatomic measurements of femoral articular cartilage thickness in seven knees

\begin{tabular}{lllr}
\hline Measurement & Measuring point & ICC $(95 \%$ Cl) & p Value \\
\hline FAC thickness & Lateral condyle & $0.795(-0.097$ to 0.964$)$ & 0.016 \\
& Medial condyle & $0.883(0.045$ to 0.981$)$ & $<0.001$ \\
& Intercondylar notch & $0.732(-0.46$ to 0.954$)$ & 0.071
\end{tabular}

FAC, femoral articular cartilage, ICC, intraclass correlation coefficient.

available US in measuring cartilage thickness in order to apply this technique in clinical practice and multicentre studies.

Our results demonstrated a high interexaminer reliability of US acquisition and measurement of FAC thickness between 10 expert sonographists. Intraexaminer reliability was also high for FAC thickness at the MC and acceptable at the LC and IN.

US is considered to be a highly operator-dependent technique. The main variability in evaluating articular cartilage is related to inadequate inclination or positioning of the transducer, which produces interface and/or cartilage thickness artefacts. We used suprapatellar transverse US scans because they allow an easier identification of anatomical reference points. We conducted the study according to a strict standardised scanning and measuring protocol. These factors probably contributed to the high inter/ intraexaminer reliability obtained in this study.

The accuracy or criterion validity of any method of in vivo measurements can only be assessed if we have a gold standard, ideally the anatomic specimen itself. Since the high number of cartilage thickness measurements required for our protocol had not been possible in live subjects, we chose cadaver knees for this study. Although postmortem changes could influence cartilage properties, the US images obtained from the cadaver knees were identical to those visualised in live subjects. It is possible that the preparation method used on the cartilage specimens after knee dissection could explain the slightly higher FAC thickness found in anatomic specimens with respect to US images.

The mean and range of our US and anatomic FAC thickness measures were lower than those described in healthy subjects ${ }^{916}$ probably because the specimens were from older individuals and they presented variable grades of FAC lesion.

We found a highly significant agreement between the US measures and the corresponding specimen measures of FAC thickness at both condyles after excluding from the analysis the one knee with a severe FAC lesion. This level of agreement was similar to those reported in comparative studies between threedimensional high-resolution MRI and histomorphometric knee cartilage thickness measurement in an animal model of osteoarthritis ${ }^{26}$ and between in vitro experimental $25 \mathrm{MHz}$ US and histological human FAC thickness measurement. ${ }^{25}$ Our level of agreement was better at the MC than at the LC. Agreement at the IN was not significant, probably because of the low number of knees included in the study. McCune et $a l^{14}$ used US to investigate FAC in patients treated with total knee arthroplasty. In keeping with our results, these authors described the difficulty of measuring FAC thickness at sites of cartilage damage where the cartilage-bone and the cartilagesoft tissue interfaces appeared ill defined. Thus, US measurement of FAC thickness seems to be inaccurate for severely damaged FAC. Since drug interventions that could inhibit structural damage in OA are mainly indicated in patients with mild or moderate stage of the disease, ${ }^{27}$ US seems to be a promising and accurate method for monitoring potential cartilage response to drug therapy in OA. 
We studied the articular cartilage of the femoral condyles because of the high prevalence of knee $\mathrm{OA}$ and because, as had been reported in previous studies, we were able to visualise a great part of the weight-bearing articular cartilage of the femoral condyles from a suprapatellar approach by hyperflexing the knee. ${ }^{12} 1628$ However, the posterior weight-bearing femorotibial cartilage was hidden by the patella even with a maximal knee flexion of $140^{\circ}$. In fact, a limitation of US assessment of articular cartilage is that a variable portion of it is occult to the ultrasound beam because of the bones in the joints. In addition, some patients with OA may be unable to flex their knee to $125^{\circ}$, especially those with knee synovitis and/or severe degenerative changes. Further longitudinal studies should demonstrate that US evaluation of a portion of articular cartilage is sensitive enough to change to monitor disease progression and therapy response in a given joint.

In conclusion, commercially available US demonstrated a good reproducibility in FAC thickness measurement by multiple examiners. In addition, US FAC thickness measurement was accurate in normal to moderately damaged cartilage. We suggest the potential use of US for in vivo assessment of knee articular cartilage and monitoring of disease progression and response to therapy in multicentre studies with large cohorts of patients with knee OA.

Acknowledgements: We thank Javier Gálvez, Emerging Market Modality Leader, GE Healthcare CS Ultrasonidos, for his technical support.

Funding: This study was partially supported by grants from the Spanish Ministry of Health (FISS 070499) and by Zambon, Almirall and Sanofi Aventis.

Competing interests: None declared.

\section{REFERENCES}

1. Blackburn WD, Chivers S, Bernreuter W. Cartilage imaging in osteoarthritis. Semin Arthritis Rheum 1996;25:273-81.

2. Raynauld JP, Martel-Pelletier J, Berthiaume MJ, Labonté F, Beaudoin G, de Guise $\mathrm{JA}$, et al. Quantitative magnetic resonance imaging evaluation of knee osteoarthritis progression over two years and correlation with clinical symptoms and radiologic changes. Arthritis Rheum 2004;50:476-87.

3. Amin S, LaValley MP, Guermazi A, Grigoryan M, Hunter DJ, Clancy M, et al. The relationship between cartilage loss on magnetic resonance imaging and radiographic progression in men and women with knee osteoarthritis. Arthritis Rheum 2005; 52:3152-9.

4. Drape JL, Pessis E, Auleley GR, Chevrot A, Dougados M, Ayral X. Quantitative MR imaging evaluation of chondropathy in osteoarthritic knees. Radiology 1998;208:49-55.

5. Loeuille D, Olivier P, Mainard D, Gillet P, Blum A. Magnetic resonance imaging of normal and osteoarthritic cartilage. Arthritis Rheum 1998;41:963-75.

6. Burgkart R, Glaser C, Hyhlik-Durr A, Englmeier KH, Reiser M, Eckstein F. Magnetic resonance imaging-based assessment of cartilage loss in severe osteoarthritis: accuracy, precision and diagnostic value. Arthritis Rheum 2001;44:2072-7.

7. Raynauld JP, Kauffmann C, Beaudoin G, Berthiaume MJ, de Guise JA, Bloch DA, et al. Reliability of a quatification imaging system using magnetic resonance images to measure cartilage thickness and volume in human normal and osteoarthritic knees. Osteoarthritis Cartilage 2003;11:351-60.

8. Peterfy CG, Guermazi A, Zaim S, Tirman PF, Miaux Y, White D, et al. Whole organ magnetic resonance imaging score (WORMS) of the knee in osteoarthritis. Osteoarthritis Cartilage 2004:12:177-90.

9. Grassi W, Filippucci E, Farina A. Ultrasonography in osteoarthritis. Semin Arthritis Rheum 2005;34(Suppl 2):19-23.

10. Felson DT, Zhang Y. An update on the epidemiology of knee and hip osteoarthritis with a view to prevention. Arthritis Rheum 1998;41:1343-55.

11. Carmona L, Ballina J, Gabriel R, Laffon A, EPISER Study Group. The burden of musculoskeletal diseases in the general population of Spain: results from a national survey. Ann Rheum Dis 2001;60:1040-5.

12. Aisen AM, McCune WJ, MacGuire A, et al. Sonographic evaluation of the cartilage of the knee. Radiology 1984;153:781-4.

13. Hammer M, Mielke H, Wagener P, Schwarzrock R, Giebel G. Sonography and NMR imaging in rheumatoid gonarthritis. Scan J Rheumatol 1986;15:157-64.

14. McCune WJ, Dedrick DK, Aisen AM, MacGuire A. Sonographic evaluation of osteoarthritic femoral condylar cartilage. correlation with operative findings. Clin Orthop Rel Res 1987;254:230-5.

15. Jonsson K, Buckwalter K, Helvie M, Niklason L, Martel W. Precision of hyaline cartilage thickness measurements. Acta Radiol 1992;33:234-9.

16. Martino F, Ettorre GC, Angelelli G, et al. Validity of ecographic evaluation of cartilage in gonarthrosis. Preliminary report. Clin Rheumatol 1993;12:178-83.

17. Castriota-Scanderberg A, De Micheli V, Scarale MG, Bonetti MG, Cammisa M. Precision of sonographic measurement of articular cartilage: inter- and intraobserver analysis. Skeletal Radiol 1996;25:545-9.

18. Mathiesen 0, Konradsen L, Torp-Pedersen S, Jørgensen U. Ultrasonography and articular cartilage defects in the knee: an in vitro evaluation of the accuracy of cartilage thickness and defect size assessment. Knee Surg Sports Traumatol Arthrosc 2004; 12:440-3

19. Iagnocco A, Coari G, Zoppini A. Sonographic evaluation of femoral condylar cartilage in osteoarthritis and rheumatoid arthritis. Scand J Rheumatol 1992;21:201-3.

20. Iagnocco A, Coari G. Usefulness of high resolution US in the evaluation of effusion in osteoarthritic first carpometacarpal joint. Scand J Rheumatol 2000;29:170-3.

21. Naredo E, Cabero FJ, Palop M, Collado P, Cruz A, Crespo M. Ultrasonographic findings in knee osteoarthritis: a comparative study with clinical and radiographic assessment. Osteoarthritis Cartilage 2005;13:568-74.

22. DAgostino MA, Conaghan P, Le Bars M, Baron G, Grassi W, Martin-Mola E, et al. EULAR report on the use of ultrasonography in painful knee osteoarthritis. Part 1: prevalence of inflammation in osteoarthritis. Ann Rheum Dis 2005;64:1703-9.

23. De Miguel Mendieta E, Cobo Ibáñez T, Uson Jaeger J, Bonilla Hernán G, Martin Mola E. Clinical and ultrasonographic findings related to knee pain in osteoarthritis. Osteoarthritis Cartilage 2006;14:540-4.

24. Keen HI, Wakefield RJ, Grainger AJ, Hensor EMA, Emery P, Conaghan PG. Can ultrasonography improve on radiographic assessment in osteoarthritis of the hand? A comparison between radiographic and ultrasonographic detected pathology. Ann Rheum Dis 2008;67:1116-20.

25. Myers SL, Dines K, Brandt DA, Brandt KD, Albrecht ME. Experimental assessment by high frequency ultrasound of articular cartilage thickness and osteoarthritic changes. J Rheumatol 1995;22:109-16.

26. Bolbos R, Benoit-Cattin H, Langlois JB, Chomel A, Chereul E, Odet C, et al. Measurement of knee cartilage thickness using MRI: a reproducibility study in a meniscectomized guinea pig model of osteoarthritis. NMR Biomed 2007;21:366-75

27. Jordan KM, Arden NK, Doherty M, Bannwarth B, Bijlsma JWJ, Dieppe P, et al. EULAR recommendations 2003: an evidence based approach to the management of knee osteoarthritis. Report of a Task Force of the Standing Committee for International Clinical Studies Including Therapeutic Trials (ESCISIT). Ann Rheum Dis 2003;62:1145-55.

28. Martel W, Adler RS, Chan K, Niklason L, Helvie MA, Jonsson K. Overview: New methods in imaging osteoarthritis. J Rheumatol 1991;18:32-7. 


\section{Ultrasound validity in the measurement of knee cartilage thickness}

E Naredo, C Acebes, I Möller, F Canillas, J J de Agustín, E de Miguel, E Filippucci, A lagnocco, C Moragues, R Tuneu, J Uson, J Garrido, E Delgado-Baeza and I Sáenz-Navarro

Ann Rheum Dis 2009 68: 1322-1327 originally published online August 6, 2008

doi: 10.1136/ard.2008.090738

Updated information and services can be found at:

http://ard.bmj.com/content/68/8/1322

These include:

References This article cites 28 articles, 4 of which you can access for free at: http://ard.bmj.com/content/68/8/1322\#BIBL

Email alerting service

Receive free email alerts when new articles cite this article. Sign up in the box at the top right corner of the online article.

Topic Articles on similar topics can be found in the following collections

Collections

Clinical diagnostic tests (1279)

Radiology (1112)

Radiology (diagnostics) (750)

\section{Notes}

To request permissions go to:

http://group.bmj.com/group/rights-licensing/permissions

To order reprints go to:

http://journals.bmj.com/cgi/reprintform

To subscribe to BMJ go to:

http://group.bmj.com/subscribe/ 